\title{
Keratometer: Easy to Understand
}

\section{Partha Haradhan Chowdhury ${ }^{*}$, Brinda Haren Shah ${ }^{2}$ and Nripesh}

\section{Tiwari ${ }^{3}$}

1M.Optom, Associate Professor, Principal, Department of Optometry, Shree Satchandi Jankalyan Samiti Netra Prasikshan Sansthan, Pauri, Affiliated to Uttarakhand State

\section{Short Communication}

Volume 3 Special Issue 1

Received Date: September 18, 2018

Published Date: October 08, 2018

Medical Faculty, Dehradun, India

2M.Optom, Guest Lecturer, Department of Optometry, Shree Satchandi Jankalyan Samiti Netra Prasikshan Sansthan, Pauri, Affiliated to Uttarakhand State Medical Faculty, Dehradun, India

${ }^{3}$ D.Optom, General Secretary and Chief Optometrist, Department of Optometry, Shree Satchandi Jankalyan Samiti Netra Prasikshan Sansthan, Pauri, Affiliated to Uttarakhand State Medical Faculty, Dehradun, India

*Corresponding author: Partha Haradhan Chowdhury, M. Optom, Associate Professor, Principal, Department of Optometry, Shree Satchandi Jankalyan Samiti Netra Prasikshan Sansthan, Pauri, Affiliated to Uttarakhand State Medical Faculty, Dehradun, India, Email: optometrypublish@gmail.com

\section{Abstract}

This paper describes about keratometer, its principle, various types and its use.

Keywords: Helmholtz Keratometer; Javal and Schiotz Keratometer

\section{Introduction}

Keratometer is an instrument by which we can measure 2-3 mm central anterior curvature of the cornea. It is the most important instrument for prescribing contact lenses. It can measure the Base curve of the contact lens. It is based on the fact that anterior surface of the cornea acts a convex mirror and size of the image depends on the curvature of the cornea. So, image size is varied with the curvature of the cornea.

\section{Types of Keratometer}

- Helmholtz Doubling Principle Keratometer

- Javal and Schiotz Keratometer

- Original American Optical Keratometer

- Bausch and Lomb Keratometer

Helmholtz Doubling Principle Keratometer: Here, two rotating prisms are present and create an angle with each other. Each prism have parallel surface. This doubling is dependent on the angle of the two prisms.

Javal and Schiotz Keratometer: Here, Wallaston prisms are used.

Original American Optical Keratometer: Here, bi prisms are used.

Bausch and Lomb Keratometer: Here, two prisms are used, one prism is in the horizontal direction and another prism is in the vertical direction. It consist of two apertures through which whatever the rays are passing, they are undeviated.

\section{Another Types of Keratometer are}

- One Position Keratometer

- Two Position Keratometer 


\section{Open Access Journal of Ophthalmology}

One Position Keratometer: Bausch and Lomb Keratometer and Appa Swamy Keratometer come under it. It is one position keratometer because it consists of horizontal and vertical direction prism. Without rotation, horizontal and vertical meridian can be assessed.

Two Position Keratometer: Here, Wallaston prism is used. In this case, Horizontal meridian is assessed, after that Keratometer should be rotated 90 degree apart to assess another meridian.

\section{Points to Remember}

Corneal Astigmatism is diagnosed by the Keratometer. Whatever we are seeing through the Keratometer are Mires. Mires consist of three circles. At first "+" should coincide and then " - " mires should coincide. In cases of High cylinder or Keratoconus, where contact lenses is needed to fit, Extended Keratometery should be performed. The range of the Extended Keratometer is: For steep cornea: +1.25 D lens is placed in front of keratometer, so that keratometer range will be extended upto +9.00 D. For flat cornea: $-1.00 \mathrm{D}$ lens is placed in front of the keratometer, so that keratometer range will be extended upto $-6.00 \mathrm{D}$. While performing keratometry, if mires appear blur, then patient should be instructed to blink frequently [1-3].

\section{References}

1. William J Benjamin (2006) Borish's Clinical Refraction, 2nd (Edn.).

2. Theodore Grosvenor, Theodore P Grosvenor (2007) Primary Care Optometry, 5th (Edn.).

3. Stewart Duke-Elder, David Abrams (1978) DukeElder's Practice of refraction.

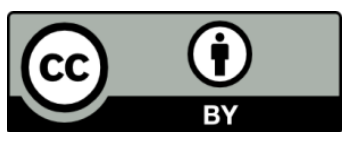

